\title{
Characterization of Desmoglein-3 Epitope Region Peptides as Synthetic Antigens: Analysis of their in vitro T-cell Stimulating Efficacy, Cytotoxicity, Stability and their Conformational Features
}

Hajnalka Szabados ${ }^{\mathrm{a}}$, Katalin Uray ${ }^{\mathrm{a}}$, Zsuzsa Majer ${ }^{\mathrm{b}}$, Pálma Sillóc ${ }^{\mathrm{c}}$, Sarolta Kárpátic ${ }^{\mathrm{c}}$ Ferenc Hudecz $^{\mathrm{a}, \mathrm{b}}$, Szilvia Bősze ${ }^{\mathrm{a} *}$

${ }^{a}$ MTA-ELTE Research Group of Peptide Chemistry, Hungarian Academy of Sciences, Eötvös Loránd University, Budapest 112, PO Box 32 H-1518, Hungary

b Department of Organic Chemistry, Institute of Chemistry, Eötvös Loránd University, Budapest, Hungary

${ }^{\mathrm{c}}$ Department of Dermatology, Venereology and Dermato-Oncology, Semmelweis University, Budapest, Hungary

\section{Short title: Desmoglein-3 epitope region peptides as synthetic antigens}

\begin{abstract}
Oligopeptides, representing B- and/or T-cell epitope(s) are promising candidates as future synthetic antigens for studying human autoimmune disorders. Synthetic peptides could be as efficient in inducing in vitro $\mathrm{T}$-cell responses as recombinant proteins. We have studied desmoglein 3 (Dsg3) peptides representing T-cell epitope regions to determine their capability to induce in vitro T-cell response and to analyze the influence of their solution conformation. The design of synthetic peptides used in this study was based on the previously described Dsg3 T-cell epitope regions and resulted in new $N$-terminally truncated versions. The peptides were prepared on solid phase and chemically characterized. In order to establish structure activity relationship, the solution conformation of the synthetic peptides has been investigated using ECD spectroscopy. The in vitro T-cell stimulating efficacy as well as in vitro cytotoxicity and stability of the peptides have been determined on peripheral blood mononuclear cells (PBMC) isolated from whole blood of Pemphigus vulgaris (PV) patients and also from healthy donors. After 20 hours stimulation the IFN- $\gamma$ content of the supernatants was measured by ELISA. Our data suggested that the in vitro IFN- $\gamma$ production of PBMC culture derived from healthy donors and PV patients induced by synthetic peptides as antigens are markedly different. Comparative analysis of in vitro activity and the capability of oligopeptides to form ordered or unordered secondary structure was performed. We measured different in vitro T-cell stimulating activity on PBMC derived from patients and
\end{abstract}


healthy donors, especially with synthetic peptides bearing high solvent sensibility which might suggest backbone flexibility.

\section{Introduction}

Desmogleins are cadherin type transmembrane adhesion proteins which form part of the core complex of desmosomes [1,2]. These cell adhesion structures are present in large amount in tissues that are exposed to intense mechanical stress, including the epidermis and the heart muscle. Desmogleins are providing adhesion by the interaction with cadherins of the opposite cell. Inside the desmosome, these proteins are attached to the intercellular plaque proteins, e.g. desmoplakin, plakoglobin and plakophilin, which in turn interact with components of the cytoskeleton [3]. Therefore, desmosomes are essential for the integrity of the specific tissues.

Desmoglein 3 (Dsg3), a member of the cadherin superfamily, is the main target of Pemphigus vulgaris (PV), a very rare but life-threatening autoimmune blistering disorder $[4,5]$. This disease affects the skin and mucous membranes and is caused by autoantibodies binding to distinct sites of the extracellular part of Dsg3, leading to gradually diminished cell-cell adhesion [6-10]. The production of pathogenic autoantibodies is organic part of the development of $\mathrm{PV}$, but several other immunological processes are required prior to autoantibody induction.

Autoantigen specific T-cells play a crucial role in the initiation and progression of PV. Activation of autoreactive $\mathrm{CD}^{4+} \mathrm{T}$-cells depends on the autoantigen presentation by specific human leukocyte antigen (HLA) class-II alleles [11-15] expressed on antigen presenting cells (APC) such as dendritic cells, macrophages, and also B-cells. There is a positive feedback between B-cells and T-cells. Dsg3-specific T-cell response provides help to the B-cells to produce Dsg3-specific autoantibodies which bind to B-cell epitope regions $[16,17]$ of the Dsg3 protein antigen.

The present, clinically applied PV diagnostics are based on autoantibody recognition of the recombinant Dsg3 antigen [18]. Characterization of the binding sites (B-cell epitope regions) of PV autoantibodies has been extensively studied [16-22]. Identification and detailed analysis of the immunodominant T-cell epitope regions of Dsg3 also have an impact in understanding the immunopathology and development of the disease and in the design of future peptide based synthetic antigens. $\mathrm{CD}^{4+} \mathrm{T}$-cells are also essential for induction of $\mathrm{CD}^{8+}$ 
cytotoxic T-cell (CTL) responses through both cytokine secretion and dendritic cell sensitization. Specific HLA class-I alleles appear to be also involved in PV which mediates natural killer cell (NK) as well as $\mathrm{CD}^{8+} \mathrm{CTL}$ function [23].

It is possible to determine T-cell epitope regions using synthetic peptides. Synthetic oligopeptides are as efficient in detecting in vitro T-cell responses as recombinant proteins. The benefit of using epitope peptides is that they do not require and probably do not go through uptake and processing, and can be presented directly by HLA class I and II molecules [24-26] via peptide loading and exchange catalyzed by a specific HLA class II molecule. HLA binding motifs with the use of peptide binding prediction methods (e.g. motif- and scoring matrix-based methods, artificial intelligence-based methods, structure-based methods) [27] may help in designing synthetic peptides, and in rationalizing their number. The fact that dominant epitopes frequently do not correspond to peptides which bind most tightly to HLA proteins [28] suggests that beside the appropriate amino acid composition for the binding motifs, the secondary structure can also affect the formation of 'HLA - synthetic peptide' complex and the recognition of this complex by the T-cell receptor.

Precise analysis of the immunodominant epitopes is important for the better understanding of the disease, and for the development of new diagnostics. There are several advantages of using synthetic epitope peptides in in vitro stimulation studies. Synthetic oligopeptides corresponding to the epitope regions of the immunodominant protein are obviously smaller molecules (from dodecamer to heptadecamer (12-17mer) synthetic peptides vs. cca 600 residues of the extracellular domain of the recombinant Dsg3 protein), therefore their production may be quicker, easier and more affordable, also the handling of the peptides is less problematic, e.g. the solubility and durability of the peptides is often better than that of larger molecules.

Different synthetic techniques have been developed to analyze the immunodominant regions of proteins. Overlapping peptide libraries are useful tools for the identification of epitope regions. Small number of peptides can be synthesized manually or with synthesizer using solid phase techniques, and in case of a large number of peptides with Pepset technique [29]. Alanine scanning is a useful tool to identify individual amino acid residues responsible for the activity of the peptide [30]. With truncated peptide libraries equimolar mixtures of $N$ - or $C$ terminally truncated T-cell epitope peptide derivatives can be tested (e.g. 15-, 14-, 13- and 12mer derivatives of the original epitope). After screening, the elements of the positive mixtures 
can be tested separately to identify the shortest amino acid sequence needed for activity, resulting in the removal of the unnecessary residues while preserving the essential ones [30]. Alanine scanning is an effective approach also for studying the role of the flanking region amino acids [31].

In some cases, the administration of oligopeptide epitopes does not elicit appropriate immune response [32]. In that case, to improve the immunogenicity of small peptides, the peptide sequence can be optimized by positional scanning, which means the substitution of an amino acid of interest with all other natural amino acids one at a time. Increase in activity identifies the preferred amino acid residues at the studied position [33].

Although the mechanisms of CTL or helper T-cell induction by soluble antigens are not fully understood, features such as solubility, stability, and the conformation of the antigen, the affinity of binding to HLA class-I or -II molecules appear to be important for the induction of T-cell responses [34-36].

Linear oligopeptides are expected to adopt multiple conformations in solution, in aqueous phase often unstructured, however, several studies to determine epitope regions using synthetic antigenic peptides assumed [35] or showed that the most potent T-cell and/or B-cell epitope peptides had tendency to form partially ordered structures in solution (authors applied various aqueous phases: water, PBS, $200 \mathrm{mM} \mathrm{NaCl}$, water/TFE 9:1 v/v and non-aqueous phases: TFE, MeOH, ACN etc.) [36-40]. For instance in aqueous solution T-cell epitope peptides bearing $\beta$-turn structural elements [36] have been found besides the $\alpha$-helical structure $[37,38]$ which was also found using the helix inducing solvent trifluoroethanol [39, 40]. These observations are suggesting that besides the linear sequence of the synthetic peptide antigen, the conformational preference in solution is also important in binding to proteins. The adopted conformation of synthetic T-cell epitope peptides in aqueous solution and the impact of conformation on the peptides' in vitro stimulating efficacy has not been studied widely.

Several theoretical and experimental tools exist for the analysis of the conformation of peptides. Using in silico predictions (e.g. Chou-Fasman secondary structure prediction, Garnier - Osguthorpe - Robson method, neural network methods (PSIPRED, JPRED), support vector machines, sequence covariation methods, etc.) the preferred conformation of the synthetic peptide can be determined [36, 41-42]. Spectroscopic methods such as proton nuclear magnetic resonance (NMR), electronic circular dichroism (ECD) or Fourier transform 
infrared spectroscopy are applicable for the characterization of the conformational preferences of peptides in solution phase [36,43-46]. All spectroscopic methods sample a collection of peptide conformations, including unfolded forms as well. Therefore, the spectra are the manifestation of the population-weighted average of the spectra characteristic for the individual contributing structures.

The combination of in silico predictions, synthetic techniques and spectroscopic methods can be powerful tool for designing synthetic peptides as future antigens representing T-cell epitope regions.

In the past, for nearly two decades, several attempts have been made to determine T-cell epitope regions of the Dsg3 protein using some, but not all of the aforementioned methods [47-50] (Table 1). Based on the HLA-DR $\beta 1 * 0402$ binding motif Wucherpfennig and colleagues identified seven peptides of the Dsg3 autoantigen [47] (Table 1). In their study in vitro T-cell response to the candidate peptides was evaluated using PBMC (peripheral blood mononuclear cells) originally isolated from peripheral blood of PV patients. From each patient, Dsg3/190-204 peptide/epitope specific T-cell lines were obtained. Two patients also showed response to peptide Dsg3/206-220, one patient responded to peptide Dsg3/251-265, and one to peptide Dsg3/762-776. T-cell response to peptides Dsg3/78-93, Dsg3/97-111 and Dsg3/512-526 has not been observed [47].

Lin et al. [48] identified T-cell response to three peptides (Dsg3/145-192, Dsg3/240-303, and Dsg3/570-614) representing stretches of the extracellular domain 1-5 of Dsg3 with the use of fusion proteins on the PBMCs of PV patients [48]. Hertl and coworkers [50] identified one immunodominant Dsg3 peptide (Dsg3/161-177; Table 1) by the stimulation of PBMCs of patients. They studied 17 mer peptides staggered by five amino acid residues [49]. Riechers et al, identified the Dsg3/380-396 peptide as another immunodominant part of the Dsg3 protein (Table 1) [50].

Moesta and coworkers [51] synthesized seven peptides and tested them for functional activity by proliferation assays using PBMC derived from PV patients. T-cell reactivity was detected to Dsg3/190-204, Dsg3/342-356, Dsg3/810-824 and Dsg3/963-974 peptides (Table 1). Their data, published as a conference abstract, also suggested that multiple epitopes may be operative in the autoimmune response in an individual patient $\left[51,63^{\text {rd }}\right.$ Annual Meeting of the Society for Investigative Dermatology, 2002, Los Angeles, CA, USA]. In the study of Veldman and coworkers Th1 and Th2 clones were established from PV patients and HLA- 
matched healthy donors [52]. The 17mer peptides used in their assay were synthesized earlier with Pepset technique and contained acetylated $\mathrm{N}$-terminus and diketopiperazine moiety at the $C$-terminus. [49]. All T-cell clones recognized the Dsg3/78-94, Dsg3/96-112, Dsg3/189-205, Dsg3/205-221 and Dsg3/250-266 peptides. Peptides Dsg3/96-112 and Dsg3/250-266 were recognized by the majority of T-cell clones from both patients and healthy donors. Peptides Dsg3/342-358 and Dsg3/376-392 were exclusively recognized only by T-cell clones from patients, while peptide Dsg3/483-499 was only recognized by T-cell clones from one healthy donor (Table 1) [52].

As this brief review of literature on this topic shows: i) PV is difficult to systematically study because of the relatively small number (4-16) of accessible donors [47-50, 52]. The difficulties are increased by stages of the disease in the different patients, different treatment, general condition of the patients, etc., ii) only few studies deal with structure - activity relationships using synthetic peptides as antigens [36-40], iii) there are no highly specific antigens related to T-cell epitope regions which could be selectively applied to distinguish in vitro T-cell response between healthy donors and PV patients [47-52], understandably, considering the different immunological, clinical status and HLA phenotype of the patients.

Based on the literature data (Table 1) we synthesized 17mer (Dsg3/189-205 and Dsg3/342358 [49]) peptides and their $N$-terminally truncated derivatives according to the Dsg3 T-cell epitope regions (Table 2). In our present study regions Dsg3/206-220 and Dsg3/762-776 [47] were represented by two new 17mer peptides (Dsg3/206-222 and Dsg3/761-777). These are elongated versions of the already published synthetic peptides [47]. In case of Dsg3/762-776 the elongation was necessary to avoid undesired side reactions on the peptide $N$ - and $C$ terminus. $\mathrm{N}$-terminally truncated derivatives of these peptides were also prepared (Table 2). The peptides were chemically characterized. The secondary structure of the selected regions of Dsg3 was predicted with Chou-Fasman algorithm, and the conformation of the $17 \mathrm{mer}$ peptides and their truncated derivatives was studied in solution by electronic circular dichroism spectroscopy (ECD). Solubility was determined in cell culture medium (aqueous phase). The in vitro cytotoxicity was determined by colorimetric tetrazolium (MTT) assay prior to the in vitro T-cell stimulating experiments. PBMCs - isolated from whole blood samples of PV patients and healthy donors - were stimulated with the synthetic peptides, and the IFN- $\gamma$ content of supernatants was determined by a two-MoAb sandwich ELISA system. A comparative analysis of these properties has to be carried out to prove the oligopeptides' applicability as synthetic antigens. 


\section{Materials and Methods}

\subsection{Chemicals and Reagents}

Solid phase peptide synthesis, analysis and purification

All amino acid derivatives and resins were purchased from Iris Biotech $\mathrm{GmBH}$ (Marktredwitz, Germany). Coupling agents, cleavage reagents and scavengers (1hydroxybenzotriazole hydrate $(\mathrm{HOBt}), \mathrm{N}, \mathrm{N}^{\prime}$-diisopropylcarbodiimide (DIC), piperidine, 1,8diazabicyclo[5.4.0] undec-7-ene (DBU), trifluoroacetic acid (TFA), thioanizole, phenol, 1,2ethanedithiol (EDT) were obtained from Sigma-Aldrich Ltd (Budapest, Hungary). 1-Methyl2-pyrrolidon was obtained from Merck Ltd (Budapest, Hungary). N,N-Dimethylformamide (DMF), dichloromethane (DCM), diethylether and dimethylsulfoxide (DMSO) were purchased from Molar Chemicals Ltd (Budapest, Hungary). Hydrochloric acid ( $\mathrm{HCl})$, acetic acid, solvent for HPLC (acetonitrile $\left(\mathrm{CH}_{3} \mathrm{CN}\right)$ ) and solvent for ECD (trifluoroethanol (TFE, NMR grade)) were purchased from Sigma-Aldrich Ltd (Budapest, Hungary).

PBMC preparation, in vitro stimulation, MTT assay and ELISA

RPMI-1640 medium, gentamicin and 3-(4,5-dimethylthiazol-2-yl)-2,5-diphenyltetrazolium bromide (MTT) were obtained from Sigma-Aldrich Ltd (Budapest, Hungary). Fetal calf serum (FCS) was purchased from Biocenter Ltd (Szeged, Hungary). Ficoll® was purchased from Amersham Biosciences (Freiburg, Germany) and Staphylococcal Enterotoxin B (SEB) was obtained from Sigma-Aldrich Ltd (Budapest, Hungary). IFN- $\gamma$ ELISA kit was purchased from BD (San Jose, CA, USA). Phosphate buffered saline (0.01 M PBS, pH=7.4) was prepared by dissolving 150 mmole $\mathrm{NaCl}, 8$ mmole $\mathrm{Na}_{2} \mathrm{HPO}_{4} \times 2 \mathrm{H}_{2} \mathrm{O}, 2.7$ mmole $\mathrm{KCl}$ and 15 mmole $\mathrm{KH}_{2} \mathrm{PO}_{4}$ (products of Sigma-Aldrich Ltd, Budapest, Hungary) in $1000 \mathrm{~mL}$ of deionized water. Tween-20 and $o$-phenylenediamine $/ \mathrm{H}_{2} \mathrm{O}_{2}$ (SigmaFast) were also purchased from Sigma-Aldrich Ltd (Budapest, Hungary). 96-well U-bottom microtest plates and 96-well flat-bottomed plates were the products of Sarstedt Ltd (Budapest, Hungary), and Nunc 96well microtest plates were obtained from Sigma-Aldrich Ltd (Budapest, Hungary).

\subsection{Synthesis of Dsg3 T-cell Epitope Region Peptide Series}

Potential T-cell epitope peptides from the Dsg3 protein sequence and their $N$-terminally truncated derivatives (Table 2) have been synthesized by solid phase peptide synthesis method 
using standard Fmoc/tBu chemistry on Rink Amide MBHA resin (nominal loading: 0.36 mmole/g). We used $t$ Bu (Thr, Ser, Tyr), OtBu (Asp, Glu), Trt (Cys, His, Gln, Asn), Pbf (Arg) and Boc (Lys, Trp) as side chain protecting groups. The Fmoc $\alpha$-amino protecting group was removed with $2 \%$ piperidine, $2 \%$ DBU/DMF (v/v) in $2+2+5+10$ minutes. Then the resin was washed five times with DMF and twice with methanol. Coupling was performed with five equivalent of Fmoc-AA/DIC/HOBt 1:1:1 (n/n/n) in NMP for $60 \mathrm{~min}$ and monitored with ninhydrin test. After completing the synthesis, the peptides from the resin and the side chain protecting groups were removed with TFA/thioanisole/phenol/water/EDT 82.5:5:5:5:2.5 $(\mathrm{v} / \mathrm{v} / \mathrm{m} / \mathrm{v} / \mathrm{v})$ for $150 \mathrm{~min}$. The crude products were lyophilized, then purified by RP-HPLC as described below, and the pure compounds were characterized by analytical RP-HPLC and ESI-MS.

\subsection{Reverse Phase High-Performance Liquid Chromatography (RP-HPLC)}

The crude products were purified on a KNAUER 2501 HPLC system (KNAUER, Berlin, Germany) using a semipreparative Phenomenex Jupiter $\mathrm{C}_{18}$ column (250 mm x $10 \mathrm{~mm}$ ) with $10 \mu \mathrm{m}$ silica (300 ̊ pore size; Gen-Lab Ltd., Budapest, Hungary). Linear gradient elution $(10-75 \% \mathrm{~B} ; 35 \mathrm{~min})$ with eluent $\mathrm{A}(0.1 \% \mathrm{TFA}$ in water $)$ and eluent $\mathrm{B}(0.1 \%$ TFA in acetonitrile $\left./ \mathrm{H}_{2} \mathrm{O}(80: 20, \mathrm{v} / \mathrm{v})\right)$ was used at a flow rate of $4 \mathrm{~mL} / \mathrm{min}$. Peaks were detected at $220 \mathrm{~nm}$. Analytical RP-HPLC was performed on an Exformma EX1600 HPLC system (GenLab Ltd., Budapest, Hungary) using an Agilent Zorbax SB-C ${ }_{18}$ column (150 mm x $4.6 \mathrm{~mm}$ ) with $5 \mu \mathrm{m}$ silica, $80 \AA$ pore size (Kromat Ltd, Budapest, Hungary) as a stationary phase. Linear gradient elution $(0-90 \% \mathrm{~B} ; 20 \mathrm{~min})$ with eluent A $(0.1 \%$ TFA in water $)$ and eluent $\mathrm{B}$ $\left(0.1 \%\right.$ TFA in acetonitrile/ $\left.\mathrm{H}_{2} \mathrm{O}(80: 20, \mathrm{v} / \mathrm{v})\right)$ was used at a flow rate of $1 \mathrm{~mL} / \mathrm{min}$. Peaks were detected at $220 \mathrm{~nm}$.

\subsection{Mass Spectrometry (MS)}

Electrospray (ESI)-mass spectrometric analysis were carried out on an Esquire 3000+ ion trap mass spectrometer (Bruker Daltonics, Bremen, Germany). Spectra were acquired in the 50 $2500 \mathrm{~m} / \mathrm{z}$ range. Samples were dissolved in a mixture of $0.1 \%$ acetic acid in acetonitrile/ $\mathrm{H}_{2} \mathrm{O}$ $(50: 50, \mathrm{v} / \mathrm{v})$.

\subsection{Amino Acid Analysis}

Amino acid analysis was performed on Sykam Amino Acid S433H analyzer (Eresing, Germany) equipped with an ion exchange separation column and postcolumn derivatization. 
Prior to analysis the peptides were hydrolyzed with $6 \mathrm{M} \mathrm{HCl}$ in sealed and evacuated tubes at $110^{\circ} \mathrm{C}$ for $24 \mathrm{~h}$. For post-column derivatization the ninhydrin method was used.

\subsection{Secondary Structure Prediction Analysis of Dsg3 T-cell Epitope Regions}

The human Dsg3 (NCBI accession number: NP_001935.2, UniProt KB: P32926) sequence used for prediction was obtained from the protein sequence database [53, 54] [http://www.ncbi.nlm.nih.gov/protein (last accessed February 2015), http://www.uniprot.org/uniprot (last accessed February 2015)].

Secondary structure of the Dsg3 protein was predicted by the Chou-Fasman prediction method [55] using 7-amino acid windows in MicroSoft Excel applying the authors' original matrices. The probability value of the predicted secondary structural element for each segment was plotted at the position of the fourth residue. Data presented in this article correspond to the Dsg3/189-205, Dsg3/206-222, Dsg3/342-358 and Dsg3/761-777 sequences, showing three additional residues on both $N$ - and $C$-termini.

2.7. Secondary Structure Determination of Dsg3 Peptides by Electronic Circular Dichroism (ECD)

ECD spectra were recorded using a Jasco 810 polarimeter (Jasco Corporation, Japan) in the $\lambda$ $=180-300 \mathrm{~nm}$ wavelength range using a $0.02 \mathrm{~cm}$ path length quartz cell at room temperature, under constant nitrogen flush with continuous scanning mode. All spectra reported here were obtained as an average of five individual scan and corrected with the solvent ECD spectra. The polarimeter was calibrated with ammonium $\alpha$-10-camphor-sulfonate. The samples were dissolved in trifluoroethanol (TFE), distilled water and a 1:1 (V/V) mixture of TFE and distilled water. The solution concentration was in the range of $0.5-0.7 \mathrm{mg} / \mathrm{mL}$ for peptide amides. ECD band intensities are expressed in molar ellipticity $\left((\theta)_{\mathrm{MR}}\right.$ in $\mathrm{deg} \mathrm{cm}^{2} / \mathrm{dmol}$ where $\mathrm{M}_{\mathrm{R}}=$ mean residue). The percentage of the different conformational elements contributing to the spectra was calculated by the Yang method [56].

\subsection{PV Patients and Healthy Donors}

Citrated/heparinated blood samples (14-18 mL) were obtained from three PV patients (Table 3) who were seen at the Department of Dermato-Venereology and Skin Oncology, Semmelweis University (Budapest, Hungary) and from three healthy control donors. The number of ethical permission for this study is: TUKEB 74-75/1998. Patients have been 
characterized as Dsg3 positive according to the results of Mesacup Dsg-3 (MB-RG7685ECD) ELISA which contains recombinant protein antigen (MBL CO., LTD, Naka-ku, Nagoya, Japan). The clinical diagnosis of PV was confirmed by direct and indirect immunofluorescence microscopy. However, the patients were in slightly different stages of the disease with different symptoms.

\subsection{Stability Studies on the Synthetic Peptides in RPMI-1640 Cell Culture Medium}

Prior to in vitro studies the stability of the synthetic peptides were determined in serum-free RPMI-1640 medium. Peptides in $1 \mathrm{mg} / \mathrm{mL}$ concentration were incubated in the medium at $37^{\circ} \mathrm{C}$ for 48 hours. Samples were analyzed with analytical RP-HPLC after 0, 24 and 48 hours of incubation using the KNAUER 2501 HPLC system (KNAUER, Berlin, Germany) with Phenomenex Jupiter $\mathrm{C}_{18}$ analytical column $(250 \mathrm{~mm}$ x $4.6 \mathrm{~mm}, 5 \mu \mathrm{m}$ silica, $300 \AA$ pore size; Gen-Lab Ltd., Budapest, Hungary). Linear gradient elution (10-75\% B; 35 min) with eluent A $\left(0.1 \%\right.$ TFA in water) and eluent $\mathrm{B}\left(0.1 \%\right.$ TFA in acetonitrile/ $\left.\mathrm{H}_{2} \mathrm{O}(80: 20, \mathrm{v} / \mathrm{v})\right)$ was used at a flow rate of $1 \mathrm{~mL} / \mathrm{min}$. Peaks were detected at $\lambda=220 \mathrm{~nm}$.

\subsection{Determination of the in vitro Cytotoxic Effect of the Peptides Using MTT Assay}

In vitro cytotoxic effect of the T-cell epitope region peptides was determined by MTT assay $[57,58]$. PBMCs of a healthy control were divided into 96-well round-bottomed plate in 100 $\mu \mathrm{L}$ RPMI-1640 culture medium with an initial cell number of $2 \times 10^{5}$ cells/well. After overnight incubation of the cells $\left(37^{\circ} \mathrm{C}\right)$ the peptides were dissolved in fresh culture medium and were added to the cells at 0.5, 5.0, 50, and $500 \mu \mathrm{M}$ concentrations. After overnight incubation at $37^{\circ} \mathrm{C}$, cells were washed twice with serum-free medium, then the cell viability was determined by MTT assay using $45 \mu \mathrm{L}$ of MTT solution (3-(4,5-dimethylthiazol-2-yl)2,5-diphenyltetrazolium bromide, $\mathrm{c}=2.0 \mathrm{mg} / \mathrm{mL}$ ) in each well. The respiratory chain $[57,58]$ and other electron transport systems of the living cell reduce MTT and thereby form nonwater-soluble violet formazan crystals within the cell; the amount of the formazan crystals can be determined spectrophotometrically. After $4.5 \mathrm{~h}$ incubation, cells were centrifuged for $5 \mathrm{~min}$ at $900 \mathrm{~g}$, than the supernatant was removed. Crystals were dissolved in DMSO, and the optical density (OD) of the samples was measured with iEMS MF ELISA reader (Labsystem, Helsinki, Finland) at $\lambda=540 \mathrm{~nm}$, and $\lambda=620 \mathrm{~nm}$ as reference wavelength. The percent of cytotoxicity was calculated using the following equation:

$$
\text { Cytotoxicity\% }=\left[1-\left(\mathrm{OD}_{\text {treated }} / \mathrm{OD}_{\text {control }}\right)\right] \times 100,
$$


where $\mathrm{OD}_{\text {treated }}$ and $\mathrm{OD}_{\text {control }}$ correspond to the optical densities of treated and control cells, respectively. Cytotoxicity\% was plotted as a function of concentration fitted to a sigmoidal curve and the $50 \%$ inhibitory concentration $\left(\mathrm{IC}_{50}\right)$ value was determined from these curves.

\subsection{In vitro Stimulation of PBMC with Dsg3 T-cell Epitope Region Peptides}

PBMC were isolated from the whole blood of the donors as described [59]. Whole citrated/heparinated blood, diluted 1:2 (v/v) in RPMI-1640 incomplete media (ICM), was centrifuged on the Ficoll density gradient [59]. PBMC were washed in ICM twice and then resuspended in RPMI-1640 supplemented with 10\% fetal calf serum (FCS). PBMC were cultured at $2 \times 10^{5}$ /well in 96 -well round-bottomed plates. Peptides (freshly dissolved in ICM) were added to the cells at concentrations $25 \mu \mathrm{M}$. The IFN- $\gamma$ concentration determined from the supernatant of PBMC stimulated by RPMI-1640 medium was considered as untreated (negative) control. As nonspecific positive control, SEB was used at $5 \mu \mathrm{g} / \mathrm{mL}$ concentration. After 20 hours of incubation, supernatants were harvested and stored at $-80{ }^{\circ} \mathrm{C}$ until use. IFN$\gamma$ content was measured by sandwich ELISA [60].

\subsection{Determination of IFN- $\gamma$ Concentration from PBMC Supernatants}

Supernatant from treated PBMC was tested for IFN- $\gamma$ content. For this, a two-MoAb sandwich ELISA (Enzyme Linked Immunosorbent Assay) kit was used in accordance with the recommendation of the manufacturer [60]. As capture antibody human anti-IFN- $\gamma$ (BD 551221) was used. The IFN- $\gamma$ concentration $(\mathrm{pg} / \mathrm{mL}$ ) of PBMC supernatant was determined using biotinylated mouse anti-IFN- $\gamma$ (BD 554550) as detection antibody. After washing with $0.01 \mathrm{M}$ PBS-Tween-20 $(5 \times 5 \mathrm{~min})$ the color reaction was developed with $o$ phenylenediamine $/ \mathrm{H}_{2} \mathrm{O}_{2}$ dissolved in deionized water and measured on iEMS MF ELISA reader (Labsystem, Helsinki, Finland) at $\lambda=492 \mathrm{~nm}$ and $\lambda=620 \mathrm{~nm}$. The IFN- $\gamma$ concentration was calculated with reference to the standard curve. Detection limit was 1 $\mathrm{pg} / \mathrm{mL}$ for IFN- $\gamma$.

\section{Results and Discussion}

We have designed, synthesized and structurally characterized Dsg3 peptides representing four T-cell epitope regions (189-205 [49], 206-220 [47], 342-358 [49], 762-776 [47]) published previously in the literature. Dsg3/206-220 and Dsg3/762-776 were elongated by two amino acids based on the Dsg3 protein sequence to form the 17mer Dsg3/206-222 and Dsg3/761- 
777. In case of Dsg3/762-776 the elongation was necessary to avoid undesired side reactions on the peptide $N$ - and $C$-terminus. In addition, $N$-terminally truncated versions of all four epitope region peptides were prepared and chemically characterized (Table 2). The solubility, secondary structure in solution, as well as in vitro cytotoxicity of the peptides were determined. The in vitro T-cell stimulatory activity of these compounds was evaluated on PBMC isolated from healthy donors and PV patients. Comparative analysis was performed regarding the applicability of the oligopeptides as synthetic antigens.

\subsection{Synthesis and Chemical Characterization of T-cell Epitope Peptide Series}

All peptides were synthesized with Fmoc/tBu solid phase method using Rink Amide MBHA resin resulting in peptide amide $N$-terminus. After TFA cleavage, the crude products were purified by RP-HPLC. The characterization of the peptides by mass spectrometry and amino acid analysis confirmed the expected composition (Table 2).

\subsection{Secondary Structure Prediction Analysis of Dsg3 T-cell Epitope Regions}

In order to correlate solution conformation with in vitro biological activity, we have performed secondary structure prediction analysis of the Dsg3 regions corresponding to the 189-205, 206-222, 342-358, and 761-777 regions by Chou-Fasman approach [55].

According to the prediction analysis data Dsg3 region 189-205 has a propensity to form a $\beta$ strand in the middle of the sequence (194-199), flanked by probable $\beta$-turns on both termini (Figure 1A). The Dsg3 region 206-222 has a slight preference for ordered, mostly $\beta$-strand structure broken with a $\beta$-turn in the middle of the sequence (Figure 1B), but the differences between the different conformational elements are small. Prediction analysis of 342-358 region shows that the $N$-terminal of the sequence may adopt $\beta$-strand or with lower probability $\alpha$-helix conformation, while its $C$-terminus is probably in $\alpha$-helical structure, according to the 7 residue long stretch (349-355) showing strong preference for this structure (Figure 1C). 761-777 region is most probably involved in forming several $\beta$-turn structures in the length of this sequence with unordered structure around residue 767 (Figure 1D).

\subsection{Secondary Structure Determination of Dsg3 Peptides by Electronic Circular Dichroism}


In order to validate the results of prediction analysis we have made an effort to determine experimentally the solution conformation of peptides described above by using ECD spectroscopy.

The secondary structures of peptides and proteins in solution can be determined with ECD spectroscopy. In solution the measured ECD curve is the sum of ECD of the different conformers. ECD spectra of the different secondary structures (ordered as periodic and aperiodic; and unordered conformations) are well documented. The ECD study of the peptides in water and TFE ( $\alpha$-helix promoting and membrane mimicking solvent) can help understanding the conformational stability and the solvent-sensitivity of peptides $[75,76]$; in addition, it can be useful at structure - function investigations.

The ECD curve of the $\alpha$-helix has a broad negative band at $\lambda \sim 222 \mathrm{~nm}$ (n- $\pi^{*}$ transition), a negative band at $\lambda \sim 208 \mathrm{~nm}$ and a very intense positive band at $\lambda \sim 192 \mathrm{~nm}$ (exciton couplet, $\left.\pi-\pi^{*}\right)[61,62]$. ECD spectrum of a $\beta$-sheet has a negative band at $\lambda \sim 215 \mathrm{~nm}$ (n- $\pi^{*}$ transition) with weaker intensity and a positive band at 195-200 $\mathrm{nm}\left(\pi-\pi^{*}\right.$ transition). Different turntypes can also be detected and distinguished by their characteristic chiral contributions. The ECD spectrum of a type I or III $\beta$-turn is similar to the $\alpha$-helix spectrum (class C spectrum), but the band intensities are weaker, the $\pi-\pi^{*}$ bands are blue-shifted, while the $n-\pi^{*}$ bands are red-shifted [63]. Type II $\beta$-turns have similarity to the $\beta$-sheet spectrum, but the bands are redshifted by $5-10 \mathrm{~nm}$, and an inverse $\gamma$-turn structure is characterized by a similar ECD shape [64]. ECD spectrum with an intensive negative band around $\lambda \sim 200 \mathrm{~nm}$ and with a weak negative shoulder near $\lambda \sim 230 \mathrm{~nm}$ can be interpreted as a random coil (U-type ECD shape). Similar spectral features but with a weak positive shoulder at $\sim 230 \mathrm{~nm}$ can be identified as polyproline type II helix (PPII) [65, 66].

The conformation of Dsg3/189-205, Dsg3/206-222, Dsg3/342-358, and Dsg3/761-777 full length peptides and their truncated derivatives was characterized by ECD spectra recorded in TFE, water, and water/TFE mixture 1:1 (v/v) which solvents mimic the hydrogen bond stabilizing and destabilizing environments, respectively. ECD data are presented in Figure 2 and Table 4, 5, and in the Supplementary Figure S1-S5 and Table S1. Changes in the spectra in different solvents can give information about the solvent-sensitivity and the conformational preference of the peptides. First we will summarize the spectroscopic features of the full length peptides, followed by the description of the truncated derivatives. 
The ECD spectra of all full length peptides in TFE (Figure 2A) show a positive and a negative $\pi-\pi^{*}$ band pair around $\lambda \sim 190 \mathrm{~nm}$ and $206-208 \mathrm{~nm}$, and an $\mathrm{n}-\pi^{*}$ broad band centralized around $\lambda \sim 222 \mathrm{~nm}$, indicating the presence of ordered, helical structure, mainly $\alpha$-helix. The location and the similarity of band intensities show that there is only a small difference between the conformer population of Dsg3/206-222 and Dsg3/342-358 peptides. Both peptides show highly ordered structures in TFE (the $\alpha$-helix content is more than $50 \%$ ). In case of Dsg3/189-205 and Dsg3/761-777 peptides the intensity of all bands are much smaller; so the ratio of ordered to unordered structures is smaller ( $\alpha$-helix content is only approximately 30\%). The two unequal negative ECD bands of the peptide Dsg3/189-205 suggest the presence of $3_{10}$ helix as well (Figure 2A). Wavelength and intensity of band extrema, and intensity ratio of the negative $\pi-\pi^{*}$ and the $n-\pi^{*}$ bands are shown in Table 4.

ECD spectra of Dsg3/189-205, Dsg3/206-222 and Dsg3/342-358 peptides in water/TFE (1:1, v/v) solvent mixture (Table 4, Figure S1) can be characterized by a positive and a negative $\pi$ $\pi^{*}$ band at $\lambda \sim 190 \mathrm{~nm}$ and $\lambda \sim 205 \mathrm{~nm}$, and a broad negative band $\left(\mathrm{n}-\pi^{*}\right)$ centralized at $\lambda \sim$ $220 \mathrm{~nm}$. All bands have smaller intensities than in TFE indicating less $\alpha$-helix content. The changed ratio of bands at $\lambda \sim 220 \mathrm{~nm}$ and $\lambda \sim 205 \mathrm{~nm}$ suggests the appearance of shorter helical structures as $3_{10}$ helices in the conformer mixture [67-68]. Besides the helical structures, unordered and shorter ordered units (turns) can be observed. In case of Dsg3/761777 peptide the ECD curve main features are the following: weak positive band $(\lambda \sim 183 \mathrm{~nm})$, stronger negative $\pi-\pi^{*}$ band at $\lambda=199 \mathrm{~nm}$, and a weak but broad negative $\mathrm{n}-\pi^{*}$ band (centralized at $\lambda=228 \mathrm{~nm}$ ). This shape indicates the presence of unordered/PPII as dominant structure. These data emphasize that the secondary structures of the first three 17 mer peptides are caused not only by the structure-inducing effect of the TFE, their ordered structure is relatively stable (their "solvent resistance" is higher than that of the fourth peptide).

The ECD spectra of the peptides in water (Figure 2B, Table 4) show a broad and intensive negative band at $\lambda \sim 200 \mathrm{~nm}$ with a weak negative shoulder at $\lambda \sim 225-230 \mathrm{~nm}$, this can be interpreted mainly as unordered/PPII structure. In case of the Dsg3/206-222 and Dsg3/761777 peptides, the intensity of the band at $\lambda \sim 198 \mathrm{~nm}$ is weaker than in case of the Dsg3/189205 and Dsg3/342-358 peptides. The types of secondary structure elements in the Dsg3/189205 and Dsg3/342-358 peptides resemble mainly unordered/ PPII helix structure.

During the secondary structure evaluation of the $\mathrm{N}$-terminally truncated derivatives of Dsg3/189-205 peptide (namely, Dsg3/190-205, Dsg3/192-205) we have observed no 
significant differences in TFE compared to the full length Dsg3/189-205 peptide. The ECD curves indicated the presence of ordered, helical structure, mainly $\alpha$-helix $(\sim 30 \%)$ with unordered and $\beta$-sheet structures besides. On the other hand, based on the shape and the lower intensities of the ECD curve of Dsg3/194-205 peptide, probably the dominant secondary structure is the $3_{10}$ helix (and/or $\beta$ I(III) turn) and $\beta$-sheet conformation apart from the unordered structure. The significantly lower band intensities and the calculated low $\alpha$-helix ( $\sim 5 \%)$ content of Dsg3/194-205 peptide suggest a higher ratio of $\beta$-sheet/ $\beta$-turn and unordered structures (Table S1, Figure S2A).

In $50 \%$ TFE-water solvent mixture ECD spectra were characterized by lower intensity bands and the blue-shifted band at $\lambda_{\max }=206 \mathrm{~nm}$. These features indicate that in the conformer population unordered structural elements are present in a higher ratio, but the helical features of the spectra are also preserved (Table S1, Figure S2B). These data suggest that the solvent resistance of these peptides is relatively high; in the 50\% solvent still the ordered structure dominates. All truncated peptides show the same ECD spectrum in water (a strong negative band at $195-200 \mathrm{~nm}$ which is accompanied by a shoulder at $\lambda \sim 230 \mathrm{~nm}$ ), which reflects their unordered structure (Table S1, Figure S2C). The ECD results in TFE are in good agreement with the Chou-Fasman prediction, which shows mainly $\beta$-structures ( $\beta$-sheet and isolated $\beta$ turns) (Figure 1A).

In TFE and 50\% TFE/water mixture the conformer population of the truncated derivatives of Dsg3/206-222 and Dsg3/342-358 peptides are very similar: mainly $\alpha$-helices (in TFE 60\% and $\sim 50 \%$ ) (Table S1; Figure S3A, S4A). However, analyzing the spectra of the shortest peptide from the Dsg3/342-358 series (Dsg3/347-358) the low intensity bands and the C-type ECD shape indicate the presence of mainly $\beta$-turns (probably $\beta \mathrm{I}(\mathrm{III})$ turns) apart from unordered conformational elements.

In $50 \% \mathrm{TFE} /$ water $1: 1 \mathrm{v} / \mathrm{v}$ as solvent the ratio of the unordered conformers has increased (Table S1, FigureS3B, S4B) as expected. In water Dsg3/206-222 peptide series shows a typical U-type ECD shape which indicates that mostly the unordered structures dominate. (Table S1, FigureS3C, S4C). Peptide series Dsg3/342-358 shows similar features, but the weak negative band at $\lambda \sim 225 \mathrm{~nm}$ red shifted compared to the full length peptide. In case of Dsg3/342-358 peptide the band intensity at $\lambda \sim 200 \mathrm{~nm}$ significantly decreased. Based on the results of Shanmugam and Polaravapu [77], this type of spectrum shows a significant 
contribution of PPII-type conformation (a mixture of unordered and PPII conformations). According to the prediction data in both peptide series periodical ordered structure dominates.

Next the Dsg3/761-777 series was analyzed. In TFE the truncated derivatives of Dsg3/761777 full length peptide show lower intensity ECD spectra, compared to the intensity of the spectra of other peptide series. This fact indicates lower helical content of the conformer population: mainly $\alpha$-helical structure was observed besides unordered structure elements (Table 5, Figure 2C). The shortest Dsg3/766-777 peptide shows typical C-type curve reflecting the presence of $\beta$-turn conformers ( $\beta$-I(III) besides unordered structures. In TFEwater mixture all peptides from this series have U-type ECD curves with low intensity (Table 5 , Figure 2D) and weaker negative bands at 225-231 nm. The higher ratio of unordered conformers besides ordered conformers (mainly turns) indicates higher backbone flexibility.

The unordered structure ratio is much higher compared to any other peptide series (Table 5, Figure 2D), showing very high solvent sensitivity and the instability of the ordered structural elements. As demonstrated on Figure 2 B and D, the ECD curves of the truncated series of Dsg3/761-777 peptide recorded in TFE-water mixture are very similar to the ECD curves of all the other peptide series in water (Figure S2C, S3C, S4C, S5, Table 4, 5, S1).

These findings suggesting the presence of isolated turns are in good agreement with the ChouFasman analysis, which showed very high probability of turn structures for the Dsg3/761-777 region.

3.4. In vitro PBMC Stimulating Activity of T-cell Epitope Region Peptide Series; IFN- $\gamma$ Production

Prior to stimulation experiments the stability and in vitro cytotoxicity of the synthetic peptides were analyzed. In the RPMI-1640 cell culture media Dsg3/206-222, Dsg3/342-358, Dsg3/343-358 and Dsg3/345-358 peptides required five minutes sonication for complete solubilization. The stability of the synthetic peptides was analyzed with RP-HPLC after 0, 24 and 48 hours of incubation in RPMI-1640 medium at $37^{\circ} \mathrm{C}$. All peptides were stable at these conditions for 48 hours. As an example, analytical RP-HPLC chromatograms corresponding to the RPMI-1640 medium and the Dsg3/761-777 peptide after 0, 24 and 48 hours of incubation presented in Figure 3 show that the peptide remains intact under these conditions, for the other peptides data are not shown. 
The in vitro cytotoxic effect of the $17 \mathrm{mer}$ peptides and their $N$-terminally truncated derivatives on PBMC cells isolated from healthy blood donors was studied at various concentrations $(0.5,5.0,50$, and $500 \mu \mathrm{M})$ using MTT assay. Data are summarized in Table 2. Dsg3/342-358 and Dsg3/345-358 peptides have shown cytotoxicity in vitro at $252 \mu \mathrm{M}$ and $290 \mu \mathrm{M} \mathrm{IC}{ }_{50}$ value, respectively. The other peptides had no cytotoxic effect in vitro even at the highest concentration $(500 \mu \mathrm{M})$ after overnight incubation.

PBMCs isolated from three PV patients (Table 3) and three healthy donors (gender and age of the donors: D1: $\odot$ (female), 44y; D2: $\widehat{o}$ (male), 59y; D3: $\uparrow, 28 \mathrm{y}$ ) were stimulated with the synthetic peptides in two parallels, using $25 \mu \mathrm{M}$ peptide concentrations for $20 \mathrm{~h}$. After stimulation, supernatants were collected and the IFN- $\gamma$ concentration was measured with a two-MoAb sandwich ELISA. The IFN- $\gamma$ concentration determined from supernatant of PBMC stimulated by the culture medium was considered as negative (untreated) control. As nonspecific positive stimulating control, SEB was used (data not shown).

The selected threshold (average IFN- $\gamma$ concentration from supernatant of unstimulated PBMC of healthy donors) was $50 \mathrm{pg} / \mathrm{mL}$ IFN- $\gamma( \pm 25 \mathrm{pg} / \mathrm{mL})$ (Figure 4). We represented an average IFN- $\gamma$ concentration (at least two parallel measurements) from PBMC cultures of patients and healthy donors. Usually the ELISA measurements resulted in less than $25 \%$ standard deviation (SD) regarding the IFN- $\gamma$ levels. Approximately in one third of the cases we faced higher standard deviation than $25 \%$, which SD can occur using supernatants for IFN- $\gamma$ determination, as we experienced earlier [39]. We created a set of conditions (the threshold value for normal IFN- $\gamma$ level generated from healthy donor untreated concentration; managing the SD values with graphical emphasis) to allow representation of all ELISA data for the peptide series (Figure 4A patients, Figure 4B healthy donors).

There is a marked difference in the IFN- $\gamma$ concentration pattern of PBMC of PV patients compared to that of healthy donors after stimulation with the synthetic peptides. In the case of healthy donors (D1, D2, D3) the IFN- $\gamma$ concentration was generally under the threshold value $(50 \mathrm{pg} / \mathrm{mL} \pm 25 \mathrm{pg} / \mathrm{mL})$ with few exceptions. We measured increased level of IFN- $\gamma$ from PBMC culture of the healthy donor D1 after stimulation with Dsg3/189-205 and Dsg3/763777 peptides. Stimulation with Dsg3/210-222 and Dsg3/206-222 peptides resulted in higher IFN- $\gamma$ concentration in case of donor D3 (Figure 4B).

Peptides within Dsg3/189-205 series show ambiguous pattern of IFN- $\gamma$ concentration values on PV patients (P1, P2, P3) PBMC cultures (Figure 4A): full length Dsg3/189-205 has no 
stimulatory effect on either of the patients' PBMC; and only Dsg3/192-205 peptide resulted in IFN- $\gamma$ concentration above the threshold with all PV patient donors' cultures.

Analyzing the IFN- $\gamma$ concentration caused by stimulation with peptides from series Dsg3/206222 and Dsg3/342-358 we can summarize the following: donor P1's IFN- $\gamma$ levels were markedly above the threshold with Dsg3/206-222, Dsg3/208-222, and Dsg3/342-358, Dsg3/345-358, Dsg3/347-358 peptides (Figure 4A). In case of PV patient P2 two peptides showed similar effects: Dsg3/208-222 and Dsg3/210-222. On the other hand, truncated peptides within series Dsg3/342-358 had no effect, but the full length Dsg3/342-358 showed relatively high activity; although with high SD value. In case of PV patient P3 the peptides with the strongest stimulating activity were: Dsg3/206-222, Dsg3/342-358 and Dsg3/345-358. Dsg3/347-358 peptide has similar in vitro effect although also with high SD.

The truncated derivatives of the full length Dsg3/761-777 peptide (Dsg3/763-777, Dsg3/764777 and Dsg3/766-777) were found to be the most unambiguous series: the determined IFN- $\gamma$ was above the threshold for both P1 and P2 PV patients. In case of patient P3 we measured high IFN- $\gamma$ concentration after the stimulation with Dsg3/764-777 and moderate value with Dsg3/763-777 peptide. The in vitro activity of the full length Dsg3/761-777 was not satisfactory. It is important to note, that the truncated peptides of this series were remarkably effective on PBMC cultures of PV patients, and at the same time they were almost inactive on PBMC from healthy donors (except one minor discrepancy in case of donor D1 and Dsg3/763-777 peptide).

\section{Conclusions}

Synthetic peptides are as efficient in inducing in vitro T-cell responses as recombinant proteins. The use of peptides represents an advantage as they do not require uptake and processing, and can be presented directly by the major histocompatibility complex (MHC, human leukocyte antigen (HLA) class I and II) molecules of antigen presenting cells like monocytes, B-lymphocytes and macrophages [26-28]. The naturally processed peptides enter the intracellular endosomal processing pathway in which the bulk of the de novo synthesized HLA molecules are loaded. By contrast, synthetic peptides have limited access to this dominant intracellular processing pathway, and rely instead for their presentation on a small 
number of recirculating cytoplasmic HLA molecules (after cellular uptake) or by peptide exchange occurring at the cell surface [69-73].

The in vitro applicability of synthetic peptides as antigens depends on their properties. Besides the primary structure a synthetic antigen has to be: (i) soluble in the cell culture medium, (ii) stable in the in vitro environment, (iii) non cytotoxic on isolated PBMC culture, and according to our hypothesis (iv) in aqueous environment their solution conformation should be relatively flexible for providing efficient T-cell stimulatory ability in vitro. Our Dsg3 peptide series have been investigated in view of the above listed requirements. To define suitable synthetic peptides or peptide series (peptide set) to fulfill these challenging criteria we performed a systematic study. In this paper we report the design, synthesis, chemical and conformational characterization, and in vitro evaluation of four 17 mer peptides representing four Dsg3 T-cell epitope regions (Dsg3/189-205, Dsg3/206-222, Dsg3/342-358 and Dsg3/761-777) and their $N$-terminally truncated derivatives (Dsg3/190-205, Dsg3/192205, Dsg3/194-205, Dsg3/208-222, Dsg3/210-222, Dsg3/343-358, Dsg3/345-358, Dsg3/347358, Dsg3/763-777, Dsg3/764-777, Dsg3/766-777).

All of the synthetic peptides were stable under in vitro conditions. Most of the peptides were soluble in the RPMI-1640 cell culture medium. The solubility of the peptides, if necessary (e.g. Dsg3/342-358), might be improved by substitution with carefully selected amino acids to preserve in vitro activity. The results obtained from the MTT assay show that none of the synthetic peptides were cytotoxic in vitro (at the concentration range applied in PBMC stimulation experiments).

The conformational features of the 17mer, full length peptides (Dsg3/189-205, Dsg3/206-222, Dsg3/342-358, Dsg3/761-777) and their $N$-terminally truncated derivatives were determined in solution using ECD spectroscopy following a Chou-Fasman secondary structure prediction analysis of the studied regions. We found that 17mer peptides Dsg3/206-222 and Dsg3/342358 and their truncated derivatives had stable ordered secondary structure in TFE and water/TFE solvent mixture, based on ECD data. These findings are in agreement of the results obtained from prediction analysis of the respective sequences: the propensity to form periodical ordered structures ( $\alpha$-helix or $\beta$-sheet) was calculated by the prediction algorithm. In 50\% TFE/water we observed matching behavior: decreased helical content and increased unordered conformer ratio in the mixture of conformers. In water the unordered structures dominate in case of all peptides. The full length Dsg3/189-205 and Dsg3/761-777 peptides 
had less contribution of ordered structural elements featuring longer stretches of unordered regions according to the prediction analysis, reinforced fully in case of Dsg3/761-777 by ECD data. The Chou-Fasman prediction for these two regions shows mainly $\beta$-structures (betasheet and isolated $\beta$-turns).

The $N$-terminally truncated derivatives of Dsg3/189-205 peptide (namely, Dsg3/190-205, Dsg3/192-205) showed similar secondary structural elements to the full length Dsg3/189-205 peptide (mainly $\alpha$-helix), except the shortest Dsg3/194-205 ( $3_{10}$ helix, with unordered and $\beta$ sheet structures). In TFE-water in spite of the growth of the unordered elements, the intensity has not decreased radically. All truncated peptides adopted mostly unordered structure in water. The truncated derivatives of Dsg3/761-777 peptide have lower intensity ECD spectra in TFE than the other truncated peptide series, which observation indicates the lower ordered structure content: $\alpha$-helical structure and unordered elements. The $N$-terminal truncation caused gradually smaller ordered structure content; the shortest peptide, Dsg3/766-777 features typical C-type curve characteristics (isolated $\beta$-turn conformers like $\beta$-turn (I-III), together with unordered structure). In TFE-water mixture as well, the ratio of unordered conformers is much higher compared to any other peptide series. This fact suggests very low solvent resistance. These findings, suggesting the presence of isolated turns, are in good agreement with the Chou-Fasman analysis, which showed very high probability of turn structures for the Dsg3/761-777 region. Although all full length peptides show the prevalence of $\alpha$-helix in the helix-promoting solvent TFE, and mostly in TFE/water mixture as well, the truncated peptide series have higher tendency to adopt $\beta$-structures (either $\beta$-turn or $\beta$-strand) besides the presence of $\alpha$-helix. Comparing the four synthetic peptide series representing Tcell epitope regions, Dsg3/761-777 series has a remarkably high instability regarding the ordered structure elements, and this solvent sensibility might suggest high backbone flexibility.

The in vitro T-cell response differences (as IFN- $\gamma$ level determined from supernatants of PBMC cultures) between healthy donors and PV patients can be detected using synthetic Dsg3 peptides as antigens. We would like to highlight the in vitro stimulatory activity of three peptides on all patients and healthy donors' PBMC cultures: Dsg3/192-205, Dsg3/342-358 and Dsg3/764-777. Following the stimulation with these synthetic peptide antigens we could distinguish the PV patients from healthy donors. Considering peptide series as antigen set instead of individual peptides, results with truncated peptides from Dsg3/761-777 series showed remarkable differences following stimulation of PBMC from PV and healthy donors. 
Comparing the in vitro activity with data from secondary structure analysis we would suggest that the solvent sensibility and high backbone flexibility of the synthetic peptides can be a considerable issue for differential in vitro PBMC stimulation ability. Overall, we can conclude that carefully chosen synthetic peptides or peptide series (set), with flexible secondary structure, can be appropriate antigens for differential in vitro T-cell stimulation for PV patients and healthy donors. Based on our results it is worth considering the combination of the following peptides as synthetic antigen set as an object for further study: Dsg3/192-205, Dsg3/342-358, Dsg3/763-777 and Dsg3/764-777. 
Figure legends

Figure 1. Predicted secondary structural elements of the Dsg3 protein regions 189-205 (A), 206-222 (B), 342-358 (C) and 761-777 (D) with 3 additional amino acid residues on both $\mathrm{N}$ and $C$-termini in order to show the region in the relation of the intact protein. A sequential segment length of 7 amino acids was chosen. The probability value of the predicted secondary structural element for each segment was plotted at the position of the fourth residue.

Figure 2. The ECD spectra of peptides Dsg3/189-205, Dsg3/206-222, Dsg3/342-358, and Dsg3/761-777 recorded in TFE (A), and water (B), full length Dsg3/761-777 peptide and its $N$-terminally truncated derivatives in TFE (C), full length Dsg3/761-777 peptide and its $N$ terminally truncated derivatives in water/TFE (1:1, v/v) (D).

Figure 3. Analytical RP-HPLC chromatogram of the RPMI-1640 incomplete medium and the Dsg3/761-777 peptide after 0 hour, 24 hours and 48 hours. Peak 4 represents the Dsg3/761777 peptide while peaks 1-3 and 5-10 are components of the RPMI-1640.

Figure 4. Average IFN- $\gamma$ concentration of 2-2 parallel measurements from supernatants of three PV patients (A) and three healthy donors (B) determined by ELISA after 20 hours of in vitro PBMC stimulation with $25 \mu \mathrm{M}$ synthetic peptide. Filled symbols represent IFN- $\gamma$ levels with maximum $25 \%$ standard deviation, while unfilled symbols represent uncertain IFN- $\gamma$ levels where the standard deviation is higher than $25 \%$. The threshold value $(50 \mathrm{pg} / \mathrm{ml} \mathrm{IFN}-\gamma)$ and the $\pm 25 \mathrm{pg} / \mathrm{ml}$ represented by grey horizontal area. The full length peptides are highlighted in frames.

\section{References}

[1] Thomason HA, Scothern A, McHarg S, Garrod DR. Desmosomes: adhesive strength and signalling in health and disease. Biochem. J. 2010. 429: 419-433.

[2] Delva E, Tucker DK, Kowalczyk AP. The desmosome. Cold Spring Harb. Perspect. Biol. 2009. 1: a002543.

[3] Green KJ, Simpson CL. Desmosomes: new perspectives on a classic. J. Invest. Dermatol. 2007. 127: 2499-2515. 
[4] Stanley JR. Cell adhesion molecules as targets of autoantibodies in pemphigus and pemphigoid, bullous diseases due to defective epidermal cell adhesion. Adv. Immunol. 1993. 53: $291-325$.

[5] Amagai M. Adhesion molecules. I. Keratinocyte-keratinocyte interactions, cadherins and pemphigus. J. Invest. Dermatol. 1995. 104: 146-152.

[6] Schiltz JR, Michel B. Production of epidermal acantholysis in normal human skin in vitro by the IgG fraction from pemphigus serum. J. Invest. Dermatol. 1976. 67: 254-260.

[7] Anhalt GJ, Labib RS, Voorhees JJ, Beals TF, Diaz LA. Induction of pemphigus in neonatal mice by passive transfer of IgG from patients with the disease. N. Engl. J. Med. 1982. 306: 1189-1196.

[8] Hashimoto K, Shafran KM, Webber PS, Lazarus GS, Singer KH. Anti-cell surface pemphigus autoantibody stimulates plasminogen activator activity of human epidermal cells. J. Exp. Med. 1983. 157: 259-272.

[9] Amagai M, Kárpáti S, Prussick R, Klaus-Kovtun V, Stanley JR. Autoantibodies against the amino-terminal cadherin-like binding domain of Pemphigus vulgaris antigen are pathogenic. J. Clin. Invest. 1992. 90: 919-926.

[10] Mahoney MG, Wang Z, Rothenberger KL, Koch PJ, Amagai M, Stanley JR. Explanation for the clinical and microscopic localization of lesions in Pemphigus foliaceus and vulgaris. $J$. Clin. Invest. 1999. 103: 461-468.

[11] Scharf SJ, Long CM, Erlich HA. Sequence analysis of the HLA-DR beta and HLA-DQ beta loci from three Pemphigus vulgaris patients. Hum. Immunol. 1988. 22: 61-69.

[12] Scharf SJ, Friedmann A, Brautbar C, Szafer F, Steinman L, Horn G, Gyllensten U, Erlich HA. HLA class II allelic variation and susceptibility to Pemphigus vulgaris. Proc. Natl. Acad. Sci. USA. 1988. 85: 3504-3508.

[13] Ahmed AR, Yunis EJ, Khatri K, Wagner R, Notani G, Awdeh Z, Alper CA. Major histocompatibility complex haplotype studies in Ashkenazi Jewish patients with Pemphigus vulgaris. Proc. Natl. Acad. Sci. USA. 1990. 87: 7658-7662.

[14] Ahmed AR, Wagner R, Khatri K, Notani G, Awdeh Z, Alper CA, Yunis EJ. Major histocompatibility complex haplotypes and class II genes in non-Jewish patients with Pemphigus vulgaris. Proc. Natl. Acad. Sci. USA. 1991. 88: 5056-5060.

[15] Wucherpfennig KW, Strominger JL. Selective binding of self peptides to diseaseassociated major histocompatibility complex (MHC) molecules: a mechanism for MHClinked susceptibility to human autoimmune diseases. J. Exp. Med. 1995. 181: 1597-1601.

[16], Szabados H, Bősze Sz, Silló P, Kárpát S, Hudecz F, Uray K. The mapping of linear Bcell epitope regions in the extracellular parts of the desmoglein 1 and 3 proteins: recognition of immobilized peptides by pemphigus patients' serum autoantibodies. J. Pep. Sci. 2013. 19: 84-94. 
[17] Dworschak J, Recke A, Freitag M, Ludwig RJ, Langenhan J, Kreuzer OJ, Zillikens D, Schmidt E. Mapping of B cell epitopes on desmoglein 3 in pemphigus vulgaris patients by the use of overlapping peptides. J. Dermatol. Sci. 2012. 65: 102-109.

[18] Ishii K, Amagai M, Hall RP, Hashimoto T, Takayanagi A, Gamou S, Shimizu N, Nishikawa T. Characterization of autoantibodies in pemphigus using antigen-specific enzyme-linked immunosorbent assays with baculovirus-expressed recombinant desmogleins. J. Immunol. 1997. 159: 2010-2017.

[19] Sekiguchi M, Futei Y, Fujii Y, Iwasaki T, Nishikawa T, Amagai M. Dominant autoimmune epitopes recognized by pemphigus antibodies map to the $\mathrm{N}$-terminal adhesive region of desmogleins. J. Immunol. 2001. 167: 5439-5448.

[20] Futei Y, Amagai M, Sekiguchi M, Nishifuji K, Fujii Y, Nishikawa T. Use of domainswapped molecules for conformational epitope mapping of desmoglein 3 in Pemphigus Vulgaris. J. Invest. Dermatol. 2000. 115: 829-834.

[21] Bhol K, Natarajan K, Nagarwalla N, Mohimen A, Aokit V, Ahmed AR. Correlation of peptide specificity and IgG subclass with pathogenic and nonpathogenic autoantibodies in pemphigus vulgaris: a model for autoimmunity. Proc. Natl. Acad. Sci. U. S. A. 1995. 92: $5239-5243$.

[22] Lucchese A, Mittelman A, Tessitore L, Serpico R, Sinha AA, Kanduc D. Proteomic definition of a desmoglein linear determinant common to Pemphigus vulgaris and Pemphigus foliaceous. J. Trans. Med. 2006. 4: 37-45.

[23] Amber KT, Staropoli P, Shiman MI, Elgart GW, Hertl M. Autoreactive T cells in the immune pathogenesis of Pemphigus vulgaris. Exp. Dermatol. 2013. 22: 699-704.

[24] Gómara MJ, Haro I. Synthetic peptides for the immunodiagnosis of human diseases. Curr. Med. Chem. 2007. 14: 531-546.

[25] Ceppellini R, Frumento G, Ferrara GB, Tosi R, Chersi A, Pernis B. Binding of labelled influenza matrix peptide to HLA DR in living B lymphoid cells. Nature. 1989. 339:392-394.

[26] Watts C. Capture and processing of exogenous antigens for presentation on MHC molecules. Annu. Rev. Immunol. 1997. 15: 821-850.

[27] Liao WWP, Arthur JW. Predicting peptide binding to major histocompatibility complex molecules. Autoimmun. Rev. 2011. 10: 469-473.

[28] Carmicle S, Steede NK, Landry SJ. Antigen three-dimensional structure guides the processing and presentation of helper T-cell epitopes. Mol. Immunol. 2007. 44: 1159-1168.

[29] Veldman CM, Gebhard KL, Uter W, Wassmuth R, Grötzinger J, Schultz E, Hertl M. T cell recognition of desmoglein 3 peptides in patients with Pemphigus vulgaris and healthy individuals. J. Immunol. 2004. 172: 3883-3892. 
[30] Shimizu T, Uenishi H, Teramura Y, Iwashiro M, Kuribayashi K, Tamamura H, Fujii N, Yamagishi H. Fine structure of a virus-encoded helper T-cell epitope expressed on FBL-3 tumor cells. J. Virol. 1994. 68: 7704-7708.

[31] Caccamo N, Meraviglia S, La Mendola C, Bosze S, Hudecz F, Ivanyi J, Dieli F, Salerno A. Characterization of HLA-DR- and TCR-binding residues of an immunodominant and genetically permissive peptide of the $16-\mathrm{kDa}$ protein of Mycobacterium tuberculosis. Eur $J$ Immunol. 2004. 34: 2220-2229.

[32] Purcell AW, Zeng W, Mifsud NA, Ely LK, MacDonald WA, Jackson DC. Dissecting the role of peptides in the immune response: theory, practice and the application to vaccine design. J. Pept. Sci. 2003. 9: 255-281.

[33] Uray K, Hudecz F. Effect of core epitope modification on the antibody recognition of a MUC2 mucin peptide. Mol Divers. 2012. 16:103-112.

[34] Rammensee HG. Chemistry of peptides associated with MHC class I and class II molecules. Curr. Op. Immunol. 1995. 7: 85-96.

[35] Berkower I, Buckenmeyer GK, Berzofsky JA. Molecular mapping of a histocompatibility-restricted immunodominant $\mathrm{T}$ cell epitope with synthetic and natural peptides: implications for T cell antigenic structure. J Immunol. 1986. 136: 2498-2503.

[36] Siddiqui MZ, Sharma AK, Kumar S, Kumar A, Bhat PN. Conformation of a T-cell stimulating peptide of interleukin- $1 \beta$ protein: circular dichroism studies. Int. J. Biol. Macromol. 1994. 16: 259-263.

[37] Estaquier J, Boutillon C, Ameisen JC, Gras-Masse H, Lecocq JP, Barbier B, Dixson A, Tartar A, Capron A, Auriault C. T helper cell epitopes of the human immunodeficiency virus (HIV-1) NEF protein in rats and chimpanzees. Mol. Immunol. 1992. 29: 489-499.

[38] Partidos CD, Delmas A, Steward MW. Structural requirements for synthetic immunogens to induce measles virus specific CTL responses. Mol. Immunol. 1996. 33: 1223 1229.

[39] Bősze Sz, Caccamo N, Majer Zs, Mező G, Dieli F, Hudecz F. In vitro T-cell immunogenicity of oligopeptides derived from the region $92-110$ of the $16-\mathrm{kDa}$ protein of Mycobacterium tuberculosis. Biopolymers. 2004. 76: 467-476.

[40] Holly S, Majer Zs, Tóth GK, Váradi Gy, Rajnavölgyi É, Laczkó I, Hollósi M. Circular dichroism and Fourier-transform infrared spectroscopic studies on T-cell epitopic peptide fragments of influenza virus hemagglutinin. Biochem. Biophys. Res. Commun. 1993. 193: 1247-1254.

[41] Garnier J, Osguthorpe DJ, Robson B. Analysis of the accuracy and implications of simple methods for predicting the secondary structure of globular proteins. J. Mol. Biol., 1978. 120: $97-120$. 
[42] Garnier J, Gibrat JF, Robson B. GOR method for predicting protein secondary structure from amino acid sequence. Methods Enzymol., 1996. 266: 540-553.

[43] Du JT, Li YM, Ma QF, Qiang W, Zhao YF, Abe H, Kanazawa K, Qin XR, Aoyagi R, Ishizuka $Y$, Nemoto $T$, Nakanishi $H$. Synthesis and conformational properties of phosphopeptides related to the human tau protein. Reg. Pep. 2005. 130: 48-56.

[44] Dupakuntla M, Ghosalkar JD, Mahale SD. Characterization of peptide 20-30 of follicle stimulating hormone receptor as an antagonist of receptor activity: significance of charged residues. Chem. Biol. Drug Des. 2009. 73: 108-114.

[45] Bertrand A, Brito RM, Alix, AJP, Lancelin JM, Carvalho RA, Geraldes CFGC, LakhdarGhazal F. Conformation study of HA(306-318) antigenic peptide of the haemagglutinin influenza virus protein. Spectrochimica Acta Part A. 2006. 65: 711-718.

[46] Anderson DC, Van Schooten WC, Barry ME, Janson AA, DeVries RR. Use of flanking sequences to study secondary structure-activity correlations of a Mycobacterium leprae $\mathrm{T}$ cell epitope. Eur. J. Immunol. 1990. 20: 2691-2697.

[47] Wucherpfennig KW, Yu B, Bhol K, Monos DS, Argyris E, Karr RW, Ahmed AR, Strominger JL. Structural basis for major histocompatibility complex (MHC)-linked susceptibility to autoimmunity: charged residues of a single MHC binding pocket confer selective presentation of self-peptides in Pemphigus vulgaris. Proc. Natl. Acad. Sci. USA. 1995. 92: 11935-11939.

[48] Lin MS, Swartz SJ, Lopez A, Ding X, Fernandez-Vina MA, Stastny P, Fairley JA, Diaz LA. Development and characterization of desmoglein- 3 specific $\mathrm{T}$ cells from patients with Pemphigus vulgaris. J. Clin. Invest. 1997. 99: 31-40.

[49] Hertl M, Amagai M, Sundaram H, Stanley J, Ishii K, Katz SI. Recognition of desmoglein 3 by autoreactive $\mathrm{T}$ cells in Pemphigus vulgaris patients and normals. J. Invest. Dermatol. 1998. 110: 62-66.

[50] Riechers R, Grotzinger J, Hertl M. HLA class II restriction of autoreactive T cell responses in Pemphigus vulgaris: review of the literature and potential applications for the development of a specific immunotherapy. Autoimmunity 1999. 30: 183-196.

[51] Moesta M, Stevanovic S, Rammensee HG, Lin MS, Sinha AA. Identification of intracellular T cell epitopes in Pemphigus vulgaris. J. Invest. Dermatol. 2002. 119: 319.

[52] Veldman CM, Gebhard KL, Uter W, Wassmuth R, Grötzinger J, Schultz E, Hertl M. T Cell Recognition of Desmoglein 3 Peptides in Patients with Pemphigus Vulgaris and Healthy Individuals. J. Immunol. 2004. 172: 3883-3892 .

[53] Wheeler GN, Parker AE, Thomas CL, Ataliotis P, Poynter D, Arnemann J, Rutman AJ, Pidsley SC, Watt FM, Rees DA, Buxton RS, Magee AI. Desmosomal glycoprotein DGI, a component of intercellular desmosome junctions, is related to the cadherin family of cell adhesion molecules. Proc. Natl. Acad. Sci. U. S. A. 1991; 88: 4796-4800. 
[54] Amagai M, Klaus-Kovtun V, Stanley JR. Autoantibodies against a novel epithelial cadherin in Pemphigus vulgaris, a disease of cell adhesion. Cell 1991; 67: 869-877.

[55] Chou PY, Fasman GD. Prediction of protein conformation. Biochem. 1974; 13: 222-245.

[56] Yang JT, Wu C-SC, Martinez HM. Calculation of protein conformation from circular dichroism. Methods Enzymol. 1986. 30: 208-269.

[57] Slater TF, Sawyer B, Straeuli U. Studies on succinate-tetrazolium reductase systems. III. points of coupling of four different tetrazolium salts. Biochim. Biophys. Acta 1963. 77: 383393.

[58] Mosmann T. Rapid colorimetric assay for cellular growth and survival: application to proliferation and cytotoxicity assays. J. Immunol. Methods 1983. 65: 55-63.

[59] Jurcevic S, Hills A, Pasvol G, Davidson RN, Ivanyi J, Wilkinson RJ. T cell responses to a mixture of Mycobacterium tuberculosis peptides with complementary HLA-DR binding profiles. Clin. Exp. Immunol. 1996. 105: 416-421.

[60] Meager A, in Lymphokines and Interferons: A Practical Approach (Eds: Clemens M, Morris A, Gearing A) IRL Press Ltd, Oxford, 1987, pp. 105-127.

[61] Sathyanarayana BK, Applequist J. Theoretical $\pi-\pi^{*}$ absorption and circular dichroic spectra of $\beta$-turn model peptides. Int. J. Pept. Protein Res. 1986. 27: 86-94.

[62] Woody RW. Aromatic side-chain contributions to the far ultraviolet circular dichroism of peptides and proteins. Biopolymers 1978. 17: 1451.

[63] Hollósi M, Kovér KE, Holly A, Radics L, Fasman GD. $\beta$-turns in bridged prolinecontaining cyclic peptide models. Biopolymers 1987. 26: 1555-1572.

[64] Vass E, Majer Zs, Kohalmy K, Hollósi M. Vibrational and chiroptical spectroscopic characterization of $\gamma$-turn model cyclic tetrapeptides containing two $\beta$-Ala residues. Chirality 2010. 22: 762-771.

[65] Woody RW. Circular dichroism and conformation of unordered polypeptides. Adv. Biophys. Chem. 1992. 2: 37-79.

[66] Siligardi G, Drake AF. The importance of extended conformation and, in particular, the PPII conformation for the molecular recognition of peptides. Biopolymers 1995. 37: 281-292.

[67] Manning MC, Woody RW. Theoretical Cd studies of polypeptide helices-Examination of important electronic and geometric factors. Biopolymers 1991. 31: 569-586.

[68] Toniolo C, Polese A, Formaggio F, Crisma M, Kamphuis J. Circular dichroism spectrum of a peptide $3{ }_{10}$-helix. J. Am. Chem. Soc. 1996. 118: 2744-2745.

[69] Watts C. The exogenous pathway for antigen presentation on major histocompatibility complex class II and CD1 molecules. Nat. Immunol. 2004. 5: 685-692. 
[70] Lanzavecchia A, Watts C. Peptide partners call the tune. Nature. 1994. 371: 198-199.

[71] O'Sullivan D, Sidney J, Appella F. Characterisation of the specificity of peptide binding to four DR haplotypes. J. Immunol. 1990. 145: 1799-1808.

[72] Monji T, McCormack AL, Yates JR 3rd, Pious D. Invariant-cognate peptide exchange restores class II dimer stability in HLA-DM mutants. J. Immunol. 1994. 153: 4468-4477.

[73] Ferrante A, Anderson MW, Klug CS, Gorski J. HLA-DM mediates epitope selection by a "compare-exchange" mechanism when a potential peptide pool is available. PLoS ONE. 2008. 3: e3722.

[74] Uray K, Szabados H, Silló P, Kárpáti S, Bősze Sz. Determination of in vitro T-cell stimulating activity of Dsg3 peptide antigens on PBMC from patients with pemphigus vulgaris. In: Peptides 2012. Proceedings of the $32^{\text {nd }}$ European Peptide Symposium (Eds.: Kokotos G, Constantinou-Kokotou V, Matsoukas J.). European Peptide Society, pp. 236-237.

[75] Povey JF, Smales CM, Hassard SJ, Howard MJ. Comparison of the effect of 2,2,2trifluoroethanol on peptide and protein structure and function. J. Struct. Biol. 2007. 157: 329338.

[76] Enyedi KN, Czajlik A, Knapp K, Láng A, Majer Z, Lajkó E, Kőhidai L, Perczel A, Mező G. Development of cyclic NGR-peptides with thioether linkage: structure and dynamics determining deamidation and bioactivity. J Med Chem. 2015 Feb 3. [Epub ahead of print]

[77] Shanmugam G, Polaravapu PL. Concentration- and dehydration-dependent structural transitions in poly-L-lysine J. Mol. Structure. 2008. 890: 144-149. 